\title{
Simulation Analysis of Automobile ABS based on Artificial Neural Network
}

\author{
Banglu Chen \\ College of Automobile Engineering, Wuxi Institute of Commerce, Wuxi, Jiangsu ,China, 214153 \\ email:cblu@sohu.com
}

Keywords: ANN; ABS; the relative slip ratio

\begin{abstract}
An Artificial Neural Network (ANN) was developed to analyze the automobile Anti-locking Brake System (ABS) simulation model. The linear velocity of wheel and the angular velocity could be obtained through some applications, through them we could calculate the relative slip ratio, the relative slip ratio and the braking time were input variables, the braking press and the braking distance were output. The data used to train and verify the ANN were obtained from simulation models. Trained ANN can be used to predict output.
\end{abstract}

\section{Introduction}

Vehicle safety question is always the matter people concentrate on. But many accidents have something with braking, so ABS was developed to avoid these. The system adopted the braking press with the change of the relative slip ratio and the braking time to avoid the wheel locking.

Artificial Neural Network (ANN) is a data processing system based on the structure of biological neural system. Analysis with ANN is not like modeling and simulation, But by learning from the data generated experimentally or simulation models, ANN differ from conventional programs in their ability to learn about the system to be modeled without priori knowledge of the process variables relationship.

The objectives of this paper was to develop an ANN to analyze the simulation model using the simulation data, so that corrections can be made in braking process. This will assist in developing better process controls.

\section{Model Development}

Mathematic model of vehicle braking system include three components: the whole vehicle model, tire-road braking force model, brake model. For the convenience to study, I used a quarter of whole vehicle model.

The Whole Vehicle Model. In this model, the drag forces were neglected. I got the equations as following.

$$
\begin{aligned}
& M \dot{v}=-F_{x} \\
& I \dot{\omega}=R F_{x}-T_{b}
\end{aligned}
$$

Where $\mathrm{M}$ is a quarter of the vehicle mass, $\mathrm{v}$ is the linear velocity of the wheel, $\mathrm{F}_{\mathrm{x}}$ is the road braking force, $\mathrm{I}$ is the wheel moment of inertia, $\omega$ is the angular velocity, $\mathrm{R}$ is the radius of the wheel, $\mathrm{T}_{\mathrm{b}}$ is the braking moment.

Tire-Road Braking Force Model. The road braking force is decided by friction coefficient between tire and road $\left(\varphi_{b}\right)$ and vertical force between tire and road $\left(F_{z}\right)$. The friction coefficient is the function of the wheel slip ratio $(S)$.

$$
\begin{aligned}
& F_{x}=F_{z} \cdot \varphi_{b} \\
& \varphi_{b}=f_{(S)}
\end{aligned}
$$

Brake Model. Braking moment has something with brake's size, brake hose press and the friction coefficient of friction material. 


$$
\mathrm{T}_{b}=\left(p_{l}-p_{0}\right) \mathrm{A}_{c} \eta_{m}(\mathrm{BF}) r \rho
$$

Where $p_{1}$ is the press in the brake chamber, $p_{0}$ is the press enough to conquest the drag force of the coil, $A_{\mathrm{c}}$ is the area of brake chamber, $\eta_{\mathrm{m}}$ is the mechanical efficiency of the brake, $r$ is the radius of the brake drum, $\rho$ is the ratio of the brake chamber and the brake shoe.

Simulation model is shown as Fig.1.

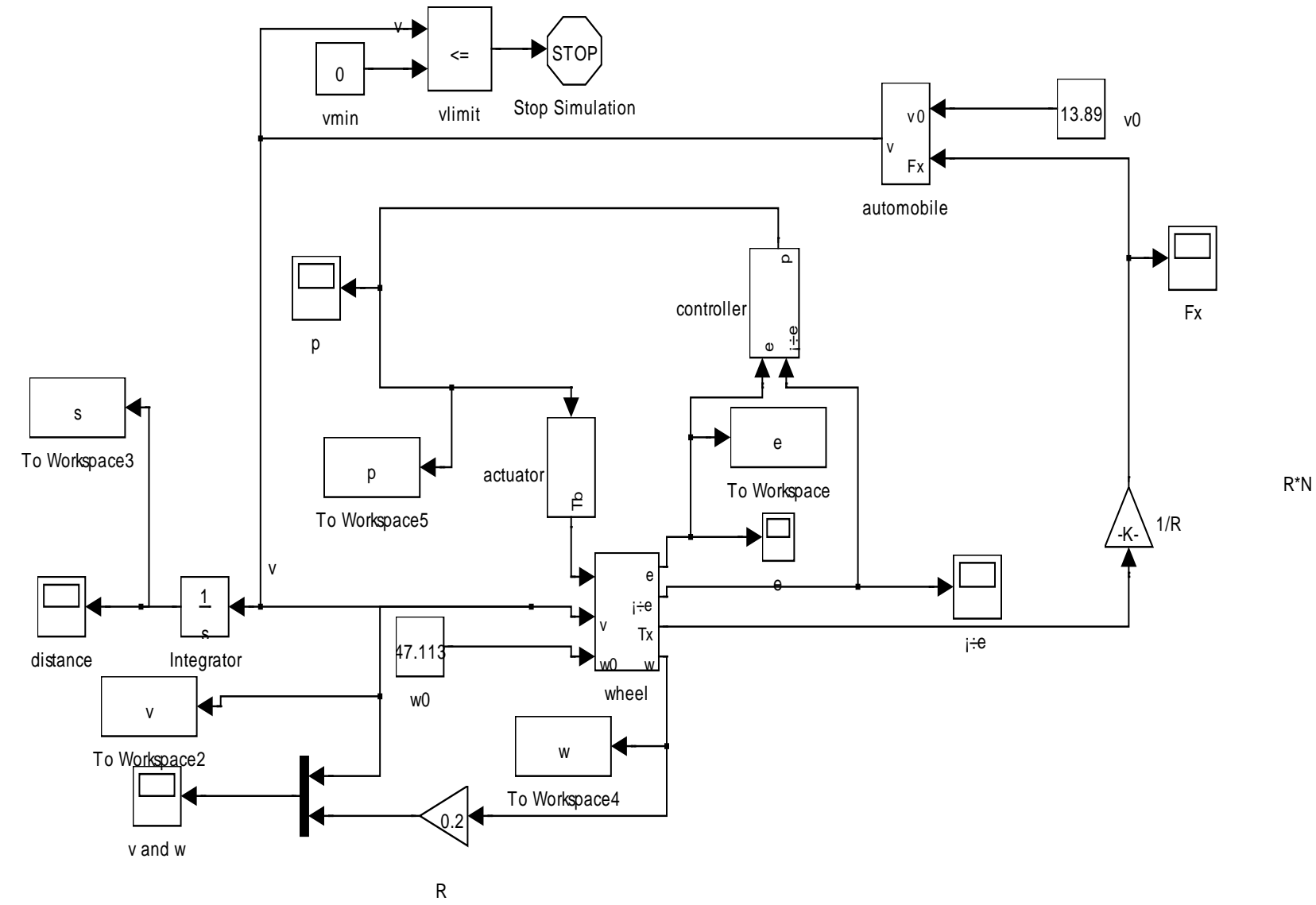

Fig.1 Simulation model

\section{Data Generation}

In the simulation model, I set the vehicle speed $50 \mathrm{~km} / \mathrm{h}$, initial press $1200000 \mathrm{pa}$, working time $0.001 \mathrm{~s}$. The data is shown as Table 1 .

Table 1 . The training and verifying data

$\begin{array}{cccccc}W & V & E & T & P & L \\ 47.113 & 13.89 & -0.19992 & 0 & 1785720 & 0 \\ 37.97373 & 13.77702 & -0.01256 & 5.76 E-06 & 974029 & 0.263031 \\ 37.60694 & 13.63766 & -0.01293 & 3.46 E-05 & 974029 & 0.52347 \\ 37.22867 & 13.49847 & -0.01306 & 0.000179 & 974029 & 0.781264 \\ 36.84643 & 13.35934 & -0.01309 & 0.000898 & 974029 & 1.036413 \\ 36.46311 & 13.22023 & -0.0131 & 0.001 & 974029 & 1.288919 \\ 36.07952 & 13.08112 & -0.0131 & 0.001 & 974029 & 1.538782 \\ 35.69586 & 12.942 & -0.0131 & 0.001 & 974029 & 1.786001 \\ 35.31218 & 12.80289 & -0.0131 & 0.001411 & 974029 & 2.030578 \\ 34.92849 & 12.66378 & -0.0131 & 0.001823 & 974029 & 2.272511 \\ 34.54481 & 12.52467 & -0.0131 & 0.002234 & 974029 & 2.511802 \\ 34.16112 & 12.38556 & -0.0131 & 0.00244 & 974029 & 2.748449 \\ 33.77744 & 12.24645 & -0.0131 & 0.002491 & 974029 & 2.982453 \\ 33.39375 & 12.10734 & -0.0131 & 0.002498 & 974029 & 3.213814 \\ 33.01007 & 11.96823 & -0.0131 & 0.002499 & 974029 & 3.442532\end{array}$




\begin{tabular}{|c|c|c|c|c|c|}
\hline 32.62638 & 11.82912 & -0.0131 & 0.0025 & 974029 & 3.668607 \\
\hline 32.2427 & 11.69001 & -0.0131 & 0.0025 & 974029 & 3.892039 \\
\hline 31.85901 & 11.5509 & -0.0131 & 0.0025 & 974029 & 4.112827 \\
\hline 31.47532 & 11.41179 & -0.0131 & 0.0025 & 974029 & 4.330973 \\
\hline 31.09164 & 11.27268 & -0.0131 & 0.0025 & 974029 & 4.546475 \\
\hline 30.70795 & 11.13357 & -0.0131 & 0.0025 & 974029 & 4.759335 \\
\hline 30.32427 & 10.99446 & -0.0131 & 0.0025 & 974029 & 4.969551 \\
\hline 29.94058 & 10.85535 & -0.0131 & 0.0025 & 974029 & 5.177124 \\
\hline 29.5569 & 10.71624 & -0.0131 & 0.0025 & 974029 & 5.382054 \\
\hline 29.17321 & 10.57713 & -0.0131 & 0.0025 & 974029 & 5.584341 \\
\hline 28.78952 & 10.43802 & -0.0131 & 0.0025 & 974029 & 5.783985 \\
\hline 28.40584 & 10.29891 & -0.0131 & 0.0025 & 974029 & 5.980986 \\
\hline 28.02215 & 10.1598 & -0.0131 & 0.0025 & 974029 & 6.175344 \\
\hline 27.63847 & 10.02069 & -0.0131 & 0.0025 & 974029 & 6.367059 \\
\hline 27.25478 & 9.881578 & -0.0131 & 0.0025 & 974029 & 6.55613 \\
\hline 26.8711 & 9.742468 & -0.0131 & 0.0025 & 974029 & 6.742558 \\
\hline 26.48741 & 9.603358 & -0.0131 & 0.002501 & 974029 & 6.926344 \\
\hline 26.10372 & 9.464248 & -0.0131 & 0.002502 & 974029 & 7.107486 \\
\hline 25.72004 & 9.325137 & -0.0131 & 0.002504 & 974029 & 7.285985 \\
\hline 25.33635 & 9.186027 & -0.0131 & 0.002508 & 974029 & 7.461841 \\
\hline 24.95267 & 9.046917 & -0.0131 & 0.002515 & 974029 & 7.635054 \\
\hline 24.56898 & 8.907806 & -0.0131 & 0.00253 & 974029 & 7.805624 \\
\hline 24.1853 & 8.768696 & -0.0131 & 0.00256 & 974029 & 7.973551 \\
\hline 23.80161 & 8.629586 & -0.0131 & 0.00262 & 974029 & 8.138835 \\
\hline 23.41792 & 8.490476 & -0.0131 & 0.002682 & 974029 & 8.301475 \\
\hline 23.03424 & 8.351365 & -0.0131 & 0.002814 & 974029 & 8.461473 \\
\hline 22.65055 & 8.212255 & -0.0131 & 0.002946 & 974029 & 8.618827 \\
\hline 22.26687 & 8.073145 & -0.0131 & 0.003076 & 974029 & 8.773538 \\
\hline 21.88318 & 7.934034 & -0.0131 & 0.003184 & 974029 & 8.925607 \\
\hline 21.4995 & 7.794924 & -0.0131 & 0.003417 & 974029 & 9.075032 \\
\hline 21.11581 & 7.655814 & -0.0131 & 0.003476 & 974029 & 9.221814 \\
\hline 20.73212 & 7.516704 & -0.0131 & 0.003491 & 974029 & 9.365953 \\
\hline 20.34844 & 7.377593 & -0.0131 & 0.003503 & 974029 & 9.507448 \\
\hline 19.96475 & 7.238483 & -0.0131 & 0.003503 & 974029 & 9.646301 \\
\hline 19.58107 & 7.099373 & -0.0131 & 0.003518 & 974029 & 9.782511 \\
\hline 19.19738 & 6.960263 & -0.0131 & 0.003533 & 974029 & 9.916077 \\
\hline 18.8137 & 6.821152 & -0.0131 & 0.003564 & 974029 & 10.047 \\
\hline 18.43001 & 6.682042 & -0.0131 & 0.003624 & 974029 & 10.17528 \\
\hline 18.04632 & 6.542932 & -0.0131 & 0.003745 & 974029 & 10.30092 \\
\hline 17.66264 & 6.403821 & -0.0131 & 0.003988 & 974029 & 10.42391 \\
\hline 17.27895 & 6.264711 & -0.0131 & 0.004473 & 974029 & 10.54426 \\
\hline 16.89527 & 6.125601 & -0.0131 & 0.005193 & 974029 & 10.66197 \\
\hline 16.51158 & 5.986491 & -0.0131 & 0.005914 & 974029 & 10.77704 \\
\hline 16.1279 & 5.84738 & -0.0131 & 0.006621 & 974029 & 10.88946 \\
\hline 15.74421 & 5.70827 & -0.0131 & 0.008962 & 974029 & 10.99924 \\
\hline 15.36053 & 5.56916 & -0.0131 & 0.009644 & 974029 & 11.10637 \\
\hline 14.97684 & 5.43005 & -0.0131 & 0.010326 & 974029 & 11.21086 \\
\hline 14.59315 & 5.290939 & -0.0131 & 0.011083 & 974029 & 11.31271 \\
\hline 14.20947 & 5.151829 & -0.0131 & 0.013295 & 974029 & 11.41192 \\
\hline 13.82578 & 5.012719 & -0.0131 & 0.015506 & 974029 & 11.50848 \\
\hline 13.4421 & 4.873608 & -0.0131 & 0.019 & 974029 & 11.6024 \\
\hline
\end{tabular}




$\begin{array}{cccccc}13.05841 & 4.734498 & -0.0131 & 0.034497 & 974029 & 11.69368 \\ 12.67473 & 4.595388 & -0.0131 & 0.038 & 974029 & 11.78231 \\ 12.29104 & 4.456278 & -0.0131 & 0.057 & 974029 & 11.86831 \\ 11.90735 & 4.317167 & -0.0131 & 0.076 & 974029 & 11.95165 \\ 11.52367 & 4.178057 & -0.0131 & 0.095 & 974029 & 12.03236 \\ 11.13998 & 4.038947 & -0.0131 & 0.114 & 974029 & 12.11042 \\ 10.7563 & 3.899837 & -0.0131 & 0.133 & 974029 & 12.18584 \\ 10.37261 & 3.760726 & -0.0131 & 0.152 & 974029 & 12.25861 \\ 9.988926 & 3.621616 & -0.0131 & 0.171 & 974029 & 12.32875 \\ 9.605241 & 3.482506 & -0.0131 & 0.19 & 974029 & 12.39623 \\ 9.221549 & 3.343396 & -0.0131 & 0.209 & 974029 & 12.46108 \\ 8.837921 & 3.204284 & -0.0131 & 0.228 & 974029 & 12.52328 \\ 8.453574 & 3.065184 & -0.01304 & 0.247 & 974029 & 12.58284 \\ 8.078746 & 2.925943 & -0.01396 & 0.266 & 974029 & 12.63976 \\ 7.701454 & 2.786738 & -0.01471 & 0.285 & 974029 & 12.69403 \\ 7.302954 & 2.647847 & -0.01308 & 0.304 & 974029 & 12.74566 \\ 6.923327 & 2.508676 & -0.01358 & 0.323 & 974029 & 12.79465 \\ 6.558581 & 2.369287 & -0.01606 & 0.342 & 974029 & 12.841 \\ 6.149757 & 2.230547 & -0.01278 & 0.361 & 974029 & 12.8847 \\ 5.768603 & 2.0914 & -0.01313 & 0.38 & 974029 & 12.92576 \\ 5.009706 & 1.813054 & -0.01457 & 0.418 & 974029 & 12.99994 \\ 4.617313 & 1.674072 & -0.0131 & 0.437 & 974029 & 13.03307 \\ 4.235045 & 1.534941 & -0.01338 & 0.456 & 974029 & 13.06356 \\ 3.850629 & 1.395842 & -0.01325 & 0.475 & 974029 & 13.0914 \\ 3.466427 & 1.256739 & -0.01314 & 0.494 & 974029 & 13.1166 \\ 3.074026 & 1.117757 & -0.01075 & 0.513 & 974029 & 13.13916 \\ 2.695431 & 0.978572 & -0.01201 & 0.532 & 974029 & 13.15907 \\ 2.307435 & 0.839525 & -0.01026 & 0.551 & 974029 & 13.17634 \\ 1.938964 & 0.70019 & -0.01636 & 0.57 & 974029 & 13.19097 \\ 1.163485 & 0.42209 & -0.01261 & 0.608 & 974029 & 13.21229 \\ 0.782539 & 0.282939 & -0.01534 & 0.627 & 974029 & 13.21899 \\ 0.042244 & 0.012761 & -0.1759 & 0.665 & 1785720 & 13.22446\end{array}$

\section{Network Training and Testing}

From the generated data (98 sets), 14 and 14 data sets were randomly selected as testing and production sets, rest 70 data sets were used for ANN training. Testing data were fed to test trained ANN after training 200 epochs. Testing errors were recorded. In the beginning of training, testing error decreased with training process. Training was continued until testing error did not decrease. If testing error was greater than the minimum testing error, the testing was continued until 200000 epochs training was reached. After training, 14 production sets were used to verify ANN performance.

Hidden Layers and Nodes. Decision of the use of number of hidden layer nodes is complex as it depends on the specific problem being solved using ANN. With too few nodes, the network may not be powerful enough for a given task. With a large number of nodes (and connections), computation is too lengthy. Sometimes an ANN may memorize the input training samples, such a network tends to perform poorly on new test samples, and is not considered to have completed learning successfully. Neural learning is considered successfully only if the system can perform well on test data which the system has not been trained. An ANN should have capabilities to generalize from input training sets, and not to memorize them. In my paper, learning rate and momentum were set 0.5 , initial weights were set 0.3 , the hidden nods were 10 , then to chose the best number of hidden layers. The result was got as Table 2. 
Table 2. Effect of numbers of hidden layers on ABS simulation errors

\begin{tabular}{l|c|c|c|c|c|c}
\hline The number of hidden layers & \multicolumn{2}{|c|}{1} & \multicolumn{2}{c|}{2} & \multicolumn{2}{c}{3} \\
\hline Output: & $P$ & $L$ & $P$ & $L$ & $P$ & $L$ \\
\hline R squared: & 1 & 0.9093 & 1 & 0.9324 & 0.9999 & 0.9332 \\
\hline r squared: & 1 & 0.9129 & 1 & 0.9345 & 1 & 0.9356 \\
\hline Mean squared error: & 5924.412 & 1.431 & 645160.1 & 1.067 & 764164.8 & 1.055 \\
\hline Mean absolute error: & 51.304 & 0.877 & 344.438 & 0.72 & 472.365 & 0.677 \\
\hline Min. absolute error: & 0 & 0 & 0 & 0 & 0 & 0.004 \\
\hline Max. absolute error: & 284.25 & 3.367 & 3597.688 & 3.117 & 3832.25 & 3.194 \\
\hline Correlation coefficient $r:$ & 1 & 0.9554 & 1 & 0.9667 & 1 & 0.9673 \\
\hline
\end{tabular}

Comparing with each other and analyzing the errors, I found that the number of hidden layer was better than any others.

After choosing the best number of hidden layer, I would choose the best nodes of hidden layer. Learning rate and momentum were set 0.5 ,initial weights were set 0.3 , I got the result as Table 3 .

Table 3. Effect of nodes in hidden layer on ABS simulation errors

\begin{tabular}{|c|c|c|c|c|c|c|}
\hline Nodes of hidden layer & \multicolumn{2}{|c|}{10} & \multicolumn{2}{|c|}{15} & \multicolumn{2}{|c|}{13} \\
\hline Output: & $P$ & $L$ & $P$ & $L$ & $P$ & $L$ \\
\hline R squared: & 1 & 0.9093 & 1 & 0.9111 & 1 & 0.9235 \\
\hline r squared: & 1 & 0.9129 & 1 & 0.9173 & 1 & 0.9276 \\
\hline Mean squared error: & 5924.412 & 1.431 & 91631.67 & 1.403 & 225454.6 & 1.207 \\
\hline Mean absolute error: & 51.304 & 0.877 & 88.623 & 0.841 & 138.424 & 0.771 \\
\hline Min. absolute error: & 0 & 0 & 0 & 0 & 0 & 0 \\
\hline Max. absolute error: & 284.25 & 3.367 & 2360.688 & 3.462 & 3913.438 & 3.368 \\
\hline Correlation coefficient r: & 1 & 0.9554 & 1 & 0.9578 & 1 & 0.9631 \\
\hline Nodes of hidden layer & \multicolumn{2}{|c|}{12} & \multicolumn{2}{|c|}{11} & \multicolumn{2}{|c|}{9} \\
\hline Output: & $P$ & $L$ & $P$ & $L$ & $P$ & $L$ \\
\hline R squared: & 1 & 0.9236 & 1 & 0.9145 & 1 & 0.9211 \\
\hline r squared: & 1 & 0.9276 & 1 & 0.9198 & 1 & 0.9259 \\
\hline Mean squared error: & 66127.72 & 1.206 & 274268.2 & 1.349 & 54180.57 & 1.245 \\
\hline Mean absolute error: & 75.571 & 0.771 & 228.425 & 0.817 & 83.862 & 0.772 \\
\hline Min. absolute error: & 0 & 0 & 0 & 0 & 0 & 0 \\
\hline Max. absolute error: & 1656.875 & 3.369 & 3617.5 & 3.454 & 1384.25 & 3.445 \\
\hline Correlation coefficient r: & 1 & 0.9631 & 1 & 0.9591 & 1 & 0.9623 \\
\hline
\end{tabular}

From the table, we could see that ANN with 10 hidden nodes provided minimum errors. The 10 hidden nodes ANN was chosen for further analysis.

Momentum and Weight. After building a frame of the network, I should decide the momentum and the weights. Value for momentum can be obtained adaptively, as in the learning rate. A well-chosen value of momentum can significantly reduce the number of iterations for convergence. Table 4 shows the effect of momentum and weights on simulation errors. Optimum combination of momentum and weight will improve the simulation. When the momentum and the weights were 0.3 , the result was worse than anyone else. So the momentum and the weights were chosen as 0.7 .

Table 4. Effect of momentum and weights on ABS simulation errors

\begin{tabular}{l|c|c|c|c|c|c}
\hline Momentum-weight & \multicolumn{2}{|c|}{$0.5-0.5$} & \multicolumn{2}{c|}{$0.7-0.7$} & \multicolumn{2}{c}{$0.9-0.9$} \\
\hline Output: & $P$ & $L$ & $P$ & $L$ & $P$ & $L$ \\
\hline R squared: & 1 & 0.9294 & 1 & 0.9312 & 1 & 0.9358 \\
\hline r squared: & 1 & 0.9339 & 1 & 0.9356 & 1 & 0.9404 \\
\hline Mean squared error: & 4768.34 & 1.115 & 3985.219 & 1.086 & 89494.51 & 1.013 \\
\hline Mean absolute error: & 25.482 & 0.695 & 11.365 & 0.677 & 122.058 & 0.629 \\
\hline Min. absolute error: & 0 & 0 & 0 & 0 & 0 & 0 \\
\hline Max. absolute error: & 464.125 & 3.345 & 437.563 & 3.32 & 1400.313 & 3.244 \\
\hline Correlation coefficient $r:$ & 1 & 0.9664 & 1 & 0.9673 & 1 & 0.9697 \\
\hline
\end{tabular}




\section{Conclusion}

In this paper I built an ANN in which there were one hidden layer, ten hidden nodes, the momentum and the weights were 0.7. Analyzing ABS simulation by this ANN using simulation data is a simple, convenient and accurate method. Errors could be reduced by selecting hidden layer, hidden nodes, appropriate combination of momentum and weights carefully. In this study, the errors could be controlled well, but the training time is too long to fit the need of braking. So I must choose better simulation model to accomplish the task.

\section{References}

[1] Zhou Kongkang, "theory of automobile”, the publish of arms industry,1996

[2] Liu Haiquan, “the study of fuzzy logic ABS control”, Jiangsu University degree paper,2000.1

[3] Zhang Jixian, “Artificial neural network”, Jiangsu University textbook

[4] Li Lin, "the study of automobile anti lock braking system based on fuzzy control”, Jiangsu University degree paper,2003.6

[5]Georg F. Mauer,Gerard F. Gissinger and Yann Chamalllard, Fuzzy Logic Continuous and Quantizing Control of an ABS Braking System, SAE 940830:1033-1042 